REGARDS

SUR LEECONOMIE ALLEMAND

BULLETIN ECONOMIQUE DU CIRAC
Regards sur l'économie allemande

Bulletin économique du CIRAC

$100 \mid 2011$

Varia

\title{
La « locomotive » de l'UE
}

Isabelle Bourgeois

\section{OpenEdition}

Journals

Édition électronique

URL : https://journals.openedition.org/rea/4218

DOI : $10.4000 /$ rea. 4218

ISBN : 978-2-8218-0894-2

ISSN : 1965-0787

Éditeur

CIRAC

Édition imprimée

Date de publication : 17 mars 2011

Pagination : 9-10

ISSN : 1156-8992

Référence électronique

Isabelle Bourgeois, «La « locomotive » de l'UE », Regards sur l'économie allemande [En ligne], 100 | mars 2011, mis en ligne le 19 septembre 2011, consulté le 28 juin 2022. URL : http://

journals.openedition.org/rea/4218; DOI : https://doi.org/10.4000/rea.4218 


\section{La " locomotive" de l'UE}

Après la profonde récession de 2009, l'économie allemande retrouve un rythme de croisière ; depuis l'automne 2009, la tendance est nettement à la hausse. En 2010, le PIB a augmenté de 3,6 \% (Destatis). Certes, la neige et le froid ont légèrement ralenti la reprise à la fin de l'année, mais seulement dans les secteurs du BTP et des transports. Les exportations booment, et la consommation est en hausse.

\begin{tabular}{|c|c|c|c|c|c|c|c|}
\hline \multicolumn{8}{|c|}{ Evolution trimestrielle du PIB allemand 2009/2010 (données CVS) } \\
\hline \multicolumn{4}{|c|}{2009} & \multicolumn{4}{|c|}{2010} \\
\hline I & II & III & IV & I & II & III & IV \\
\hline \multicolumn{8}{|c|}{ Par rapport au trimestre précédent } \\
\hline$-3,4 \%$ & $0,5 \%$ & $0,7 \%$ & $0,3 \%$ & $0,6 \%$ & $2,2 \%$ & $0,7 \%$ & $0,4 \%$ \\
\hline \multicolumn{8}{|c|}{ Par rapport au même trimestre de l'année précédente } \\
\hline$-6,3 \%$ & $-6,8 \%$ & $-4,4 \%$ & $-1,3 \%$ & $2,3 \%$ & $4,3 \%$ & $3,9 \%$ & $4,0 \%$ \\
\hline
\end{tabular}

Sans avoir renoué encore avec ses performances d'avant la crise, l'économie allemande a reconquis sa dynamique de croissance. Elle est due à l'expansion de la demande mondiale, et surtout à l'imperturbable confiance à la fois des entreprises et des consommateurs. En atteste la hausse continue depuis plusieurs mois des deux composantes des indices ifo (entreprises) ou GfK (consommateurs) : évaluation de la situation présente et perspectives d'avenir. La reprise repose maintenant solidement sur deux piliers : l'export et la demande intérieure. Les exportations ont contribué à hauteur de 1,1 point de pourcentage à la croissance en 2010 - alors que leur part avait été de -2,9 points l'année d'avant (Destatis). Elles sont dynamisées principalement par la demande des économies émergentes ou des pays tiers, celle de la zone euro demeurant encore ralentie par l'impact de la crise. La principale contribution à la reprise - les deux tiers - provient de la demande intérieure.

Les investissements ont crû de 10,7\%. Les entreprises, dont les carnets de commande ne cessent de se remplir sous l'effet du regain de la demande extérieure et domestique à la fois, et qui voient s'améliorer leur taux d'utilisation des capacités, renouvellent en effet leur parc automobile, leurs installations, et même les étendent. Cette tendance est particulièrement nette dans l'industrie des biens d'investissements, le segment le plus exportateur depuis le milieu de l'année 2010 où il avait pris la relève des biens intermédiaires ; dans ce segment, le taux d'utilisation des capacités avait renoué en janvier 2011 avec le niveau traditionnel de 84 \% (Bundesbank).

Ce retour progressif à la normale se reflète dans la bonne tenue du marché de l'emploi : non seulement, il avait remarquablement résisté durant la récession, mais le nombre de demandeurs d'emploi a baissé de 9,2 \% l'an dernier pour se situer à 2,9 millions sur l'ensemble de l'année 2010 (critères OIT ; Destatis). Cette tendance se poursuit : en février 2011, le nombre de chômeurs enregistrés atteignait 3,317 millions, soit 326000 de moins qu'un an auparavant, selon l'Agence fédérale pour l'emploi qui, contrairement à Destatis, publie des données administratives. Cette décrue s'explique notamment par le recours massif, dans l'industrie, à la réserve flexible de maind'œuvre qualifiée que constituent en Allemagne les travailleurs intérimaires (voir REA 86/08). Les entreprises embauchent de plus en plus volontiers : I'Agence de Nuremberg fait état de 417000 postes à pourvoir en février, soit 120000 de plus en un an. Quant à la fédération des $\mathrm{CCl}$ allemandes (DIHK), elle annonce que les entreprises industrielles comptent créer 300000 nouveaux emplois cette année. Les prévisions de tous les experts concordent : le nombre de chômeurs devrait se situer en dessous de la barre des 3 millions en moyenne en 2011 et donc repasser en dessous de son niveau d'avant la crise, comme le rappelle par exemple l'institut DIW dans ses prévisions 2010/11. Ce sont ces perspectives qui incitent les ménages à consommer, d'autant que, avec la normalisation progressive des heures travaillées, ils voient leurs revenus en hausse, et que la convention tarifaire actuellement en vigueur dans la métallurgie (voir REA 95/10) prévoit notamment le versement de primes.

Au total, les prévisions pour 2011 sont optimistes. En décembre, la Bundesbank tablait sur une croissance de $2 \%$; en janvier, l'institut DIW sur 2,2 \% (comme le Conseil des

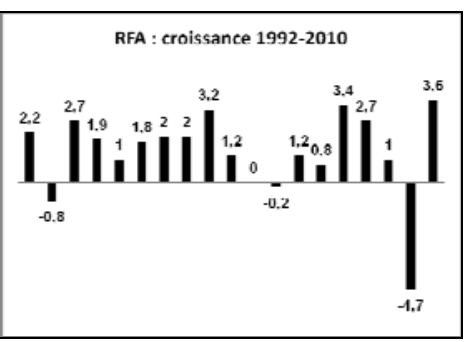

2 piliers :

export et demande intérieure

Investissements en nette hausse

Prévisions 2011 : moins de 3 millions de chômeurs ...

... et au moins $2 \%$ de croissance 
L'impact positif des réformes structurelles

La demande intérieure est indissociable des échanges
Sages en novembre), et le gouvernement fédéral sur 2,3\%. Début mars, les prévisions sont revues à la hausse, se situant désormais entre 2,5 \% (Buba) et 3,0 \% (IfW, Kiel).

Le Conseil des Sages, dont le rapport annuel, publié le 10 novembre 2010, s'intitule «Chances pour une reprise solide et durable », passe en revue les raisons qui ont permis à l'Allemagne de rebondir si vite et de faire figure aujourd'hui de «locomotive conjoncturelle » dans l'UE. Certes, les divers programmes adoptés en réaction à la crise (voir REA 89/08) ont soutenu l'économie. Bien plus importants toutefois, et surtout durables, ont été et sont toujours les effets de la politique de réformes menée par les gouvernements précédents dans trois domaines essentiels : la protection sociale, la fiscalité des entreprises et la consolidation des finances publiques. Dans le premier champ, le changement de paradigme vers une plus forte participation individuelle (loi Hartz IV, réformes du régime des retraites) a jeté les jalons d'un financement plus durable de l'Etat-Providence. Dans ce cadre, la politique conventionnelle responsable menée durant la crise par les partenaires sociaux a permis de préserver l'emploi, d'autant que la longue modération salariale et les mesures de flexibilisation du travail adoptées au cours de la décennie avaient contribué à hausser le niveau général d'activité. La réforme de la fiscalité des entreprises, achevée en 2008, avait amélioré l'attractivité du site Allemagne en comparaison internationale. Enfin, le cap de consolidation budgétaire (juste avant la crise, le déficit allemand était de $\ldots+0,1 \%$ ), puis l'adoption de critères constitutionnels pour réduire la dette, ont conforté la confiance de la finance mondiale dans la soutenabilité budgétaire de l'Allemagne. Les nouvelles dispositions s'appliquent dès cette année, afin qu'en 2016, le Bund puisse remplir l'objectif d'un déficit structurel limité à 0,35 \% du PIB. En 2011, le déficit ne doit donc pas dépasser 1,9\% du PIB. Cet objectif pourrait être atteint, l'encours fiscal ayant crû avec la reprise, et le déficit de 2010 ne se situant dès lors plus qu'à 2,2 \% au lieu des 3,5\% initialement prévus (ministère fédéral des Finances).

L'optimisme des experts se fonde sur la compétitivité du made in Germany et la vigueur d'une demande intérieure désormais en mesure d'amortir les variations de la demande mondiale. Or ces avantages ne seront durables qu'à la condition que les responsables politiques veillent à poursuivre les réformes structurelles engagées par les gouvernements précédents, ainsi que le rappelle également l'OCDE dans son rapport Allemagne 2010. II s'agit là d'une œuvre au long cours, impliquant aussi d'investir résolument dans la qualification de la main-d'œuvre et l'innovation, les deux piliers sur lesquels reposent l'emploi et la compétitivité allemande dans une économie globalisée et donc aussi la confiance des consommateurs, comme le souligne le Conseil des Sages. A plus brève échéance, il s'agit d'œuvrer à rétablir la stabilité au sein de la zone euro par une réforme du Pacte de stabilité, l'institution d'une supervision des marchés financiers au sein de l'UEM et l'adoption d'un mécanisme européen de gestion de crise qui soit certes solidaire - mais sans prévoir de soutien indifférencié, et surtout sans contrepartie, à un Etat ne respectant pas les engagements du Pacte.

S'ILS SONT CONFIANTS, LES EXPERTS N'EN SONT PAS MOINS PRUDENTS face à un certain nombre d'incertitudes sur le court terme. L'année 2011 est marquée par sept échéances électorales régionales, ce qui n'incite gère les responsables politiques à la sérénité requise pour poursuivre sur la voie des réformes. La hausse des prix alimentaires et énergétiques, mais aussi celle des importations, tire depuis ces derniers mois l'inflation qui, en Allemagne comme dans l'ensemble de la zone euro, atteint désormais le seuil des $2 \%$ au-delà duquel la BCE est appelée à intervenir. Reste l'inconnue de l'évolution des prix du pétrole.

Mais le risque principal réside dans la crise de la dette qui se manifeste dans la fragilité de la demande au sein de l'UEM, les écarts de compétitivité et la question de la soutenabilité des finances publiques. C'est ce qui avait incité la France et l'Allemagne à proposer un Pacte de compétitivité visant à renforcer la gouvernance de la zone euro. A la veille du sommet européen du 11 mars, les grandes fédérations allemandes du patronat (BDI, BDA) et des corps consulaires (DIHK, ZDH) appelaient conjointement les gouvernements à doter l'UEM d'un cadre contraignant pour tous, mais sans « pilotage bureaucratique centralisé jusque dans le détail ». Elles semblent avoir été entendues: la gouvernance de la zone euro se précise. Mais l'approche retenue est « celle de la péréquation financière entre Länder », dont les effets addictifs sont bien connus, critique le F.A.Z. (14-03). Elle ne résout en rien le problème de la compétitivité...

I. Bourgeois (14-03-2011) 